field. Such interventions could include preventive measures for infectious diseases, personal protection measures, and surveillance of personnel health markers.

Key words: diseases; evacuation; field workers; humanitarian; nongovernmental organizations (NGOs); patterns; preventive strategies; relief; trauma

E-mail: ggreenou@jhsph.edu

Prebosp Disast Med 2001;16(2):s33.

\section{Acute Hemolytic Crises in Patients with G6PD Deficiency: A Case Series \\ Dr. Anton Grunfeld}

Department of Emergency Medicine, Shaikh Khalifa Medical Center, Abu Dhabi, United Arab Emirates

Glucose-6-phosphate dehydrogenase deficiency is the most common human enzyme deficiency. It affects approximately 400 million people worldwide. Acute hemolytic crises are well-described, but uncommon presentations for patients seen in emergency departments have not been described. This paper presents a case series of five pediatric patients, four males and one female, aged from seven months to two years and ten months, who presented acutely ill and jaundiced in a general emergency department over a period of one year.

The patients' hemoglobin concentrations on presentation ranged between 37 and $50 \mathrm{~g} / \mathrm{L}$, all had evidence of acute hemolysis, and all received transfusions as part of the treatment. Ingestion of fava beans were implicated in each episode.

Key words: acute hemolytic crisis; children; clinical manifestations; emergency department; glucose-6-phosphate dehydrogenase deficiency; hemolysis; incidence

E-mail: agrunfeld@hotmail.com

Prebosp Disast Med 2001;16(2):s33.

\section{Malaria in the Emergency Room \\ E. Guévart, J. Bouquillon, M. Bessalem, E. Debecq, V. Chieux}

Service des urgences et laboratoire, Hôpital Saint Vincent, Lille, FRANCE

We observed 38 patients with 44 cases of malaria seen in the emergency room between 1997 and 1999:

1. 36 came from subsaharian Africa, only one from Asia, and one from Arabia

2. 13 were residents in their country, and 25 were back from a trip that lasted from 1 to 13 weeks

3. Half of them had taken appropriate chemoprophylaxis: most of them by chloroquine-proguanil combination

4. Symptoms appeared between 1 and 120 days after leaving the endemic area, usually within two weeks

5. Nevertheless in $25 \%$ of the cases, they appeared after more than 2 months later

6. Those patients with late reviviscence had previously taken medicine: quinine or halofantrine

7. The classic rhythmic accesses usually did not appear

8. Splenomegaly was present in $38.6 \%$ of the cases, even in case of primo-invasion

9. Three cases of pernicious accesses were observed: one of them died, another one had neuropaludism, and the last one a serious anemia

10.The diagnosis was made by blood smear examination in $38 / 44$ cases and by searching parasitic antigen (HRP) in 3 cases. It was only presumptive in the last three cases

11. The mean parasitaemia was $2 \%$, with one case at 17 and another at $25 \%$ (the last one died)

12. All patients (except for the one who died) recovered quickly with quinine or halofantrine in less than 36 hours in _ of the cases, but never more than 4 days

13. Six patients suffered a reviviscence after treatment

The main original observations concern: (1) the poor preventive activity of correct chemoprophylaxis, (2) the late apparition of symptoms, possibly until four months after leaving endemic area, particularly after previous treatment, (3) the frequency of splenomegaly in primo-invasion accesses, contrasting with the rareness of classic rhythmic accesses, and (4) the dramatic importance of rapid blood smear diagnosis.

Importance should be accorded to the single equation: "fever after leaving an endemic area $=$ malaria $=$ immediate blood smear examination» so that death by severe malaria can be avoided.

Key words: chemoprophylaxis; diagnosis; emergency department; malaria; presentation; reviviscence

Prebosp Disast Med 2001;16(2):s33.

\section{Pain in Adults Presenting to the Emergency Room: Evaluation and Treatment \\ E.Guévart; J. Bouquillon; H. Vesselier; M. Bessalem; A.M. Ramu; F.Coudou}

Service des urgencies, Hôpital Saint Vincent, Lille, FRANCE

Objective: Our objective was to to measure the frequency and the intensity of pain in patients in an emergency room, to validate different methods of measuring pain for emergencies, and to evaluate the treatment of pain in this service.

Metbods: The survey was conducted over 21 days on 983 consecutive patients. The questionnaire queried: (1) demographic data, (2) patient's own pain evaluation, (3) nurse's evaluation of the patient's pain, (4) the doctor's evaluation, (5) diagnosis classification, and (6) the nature, time, and effectiveness of the intervention against pain.

Results: The panel is similar to the usual population of this emergency room concerning the number of patients, age, gender, arrival time, and kinds of diseases. $60.3 \%$ of the patients experience pain. Medical patients and nonpainful patients are older than are surgical patients (trauma).

Using an analog visual scale (AVS), the pain reaches an average evaluation by the patients of $52.7 / 100 ; 41.7 / 100$ by the nurses; and 37.2 by the doctors. The difference between these three evaluators is highly significant statistically, but they all correlate $(r>0.60)$. Using a simple verbal scale, the average was $4.1 / 7$. It correlated with the AVS ( $r=0.61)$. The average score of the objective signs was $1.28 / 18$. It did 